\title{
MODELO PARA PROGNOSE DO CRESCIMENTO E DA PRODUÇÃO E ANÁLISE ECONÔMICA DE REGIMES DE MANEJO PARA Pinus taeda L. ${ }^{1}$
}

\author{
Fausto Weimar Acerbi Jr. ${ }^{2}$, José Roberto Soares Scolforo², Antônio Donizette de Oliveira ${ }^{2}$ e Romualdo Maestri ${ }^{3}$
}

\begin{abstract}
RESUMO - Os objetivos deste estudo foram desenvolver um sistema para prognose do crescimento e da produção de Pinus taeda L. para simular e avaliar economicamente diversos regimes de manejo, visando produzir madeira livre de nós (clearwood) e madeira para múltiplos usos; e analisar a lucratividade dos regimes de manejo em diversas condições de sítio, espaçamento, taxas de desconto e preço da madeira, considerando plantios realizados em terras da própria empresa e em terras arrendadas. O modelo desenvolvido baseia-se no conceito de compatibilidade em área basal entre o modelo para o povoamento e o modelo por classe diamétrica. Utiliza-se a distribuição Weibull, que em conjunto com os atributos do povoamento permite fazer a prognose para diferentes estratos e idades desejadas. Aplica-se então o simulador de desbaste para obter a floresta remanescente desejada. A partir desta faz-se nova prognose até a idade desejada, e novamente aplica-se o simulador de desbaste. Este procedimento é repetido até o corte final, utilizando-se para tal do software SPPpinus - Sistema de Prognose da Produção para Pinus sp. Na análise econômica foram testados dois cenários, com diversos números, épocas e intensidade de desbaste, a partir de diferentes densidades iniciais de plantio, considerando vários níveis de produtividade. Foi realizada uma análise de sensibilidade da lucratividade dos regimes de manejo gerados, considerando três taxas de desconto, dois níveis de preço da madeira e as opções de plantar Pinus sp. em terras arrendadas e em terra da própria empresa, viabilizada através da integração do SSPpinus com o software de análise de investimento Invest. Concluiu-se que o modelo de crescimento e produção desenvolvido não apresentou tendenciosidade nas estimativas, sendo, portanto, um sistema preciso; os regimes de manejo com a realização de um desbaste pré-comercial seguido de dois desbastes comerciais e desrama devem ser adotados; arrendar terras para plantar Pinus taeda é uma opção lucrativa, contudo, para o estudo em questão, o plantio de Pinus taeda em terras próprias é mais lucrativo que o plantio em terras arrendadas; a densidade inicial de plantio ideal é de 833 árvores/ha, podendo-se também adotar 1.111 árvores/ha como alternativa; e a lucratividade dos regimes de manejo aumenta bastante quando se considera a elevação do preço da madeira, devido à melhoria de sua qualidade promovida pela desrama das árvores.
\end{abstract}

Palavras-chave: Crescimento, produção, simulação, desbastes e desramas.

\section{GROWTH AND YIELD PROGNOSIS MODEL AND ECONOMIC EVALUATION OF SEVERAL MANAGEMENT REGIMES FOR Pinus taeda L.}

\begin{abstract}
This study aimed to develop a prognosis system for growth and yield of Pinus taeda L. to simulate and evaluate several management regimes for the production of multiple use clearwood to analyze the economic feasibility for several sites, spacings, and wood discount rates and prices, based on owned and rented land plantations. The developed model was based on the compatibility of stand basal area model and diametric class model. The Weibull distribution was chosen to allow the prognosis for several strata and stand ages. A thinning simulator is then applied to obtain the desired remaining stand. A new prognosis is then made and a new thinning simulation applied. This procedure is repeated up to Pinus sp. the final cutting, using the SPPinus (Prognosis
\end{abstract}

1 Recebido para publicação em 01.10.2001.

Aceito para publicação em 3.12.2001.

2 Professores do Departamento de Ciências Florestais da Universidade Federal de Lavras - UFLA - 37200-000 Lavras-MG, <fwacerbi@ufla.br>; <scolforo@ufla.br>; <donizete@ufla.br >. ${ }^{3}$ Pesquisador Sênior do Centro de Pesquisa e Tecnologia da Aracruz Celulose S.A. - 29197-000 Aracruz-ES., <rmaestri@aracruz.com.br>. 
Yield System for Pinus sp.). For the economic analysis, two scenarios were compared, using various numbers, periods and thinning intensities, based on different initial planting intensities and various yield levels. A sensitivity analysis of the economic performance was conducted for each management regime, considering three discount rate levels, two wood price levels and the option of owing or renting land. It was concluded that: the model proposed did not present bias in volume and growth estimates; the management regime with a pre-commercial followed by two commercial thinnings should be adopted; renting land for Pinus sp. plantation is economically feasible but owing land is more profitable; the initial spacing should be of 833 trees/ha; and pruning improves wood quality that increases wood price, that will eventually enhance the economic feasibility of management regimes.

Key words: Growth, yield, simulation, thinnings and prunings.

\section{INTRODUÇÃO}

Em termos de mercado mundial, as estimativas elaboradas pela FAO (Food and Agriculture Organization of the United Nations) consideram taxas médias de crescimento acima de $2,5 \%$ a.a. para o consumo de produtos da madeira até o ano 2010 (Macedo \& Mattos, 1995).

O Brasil, embora tenha se mostrado o $5^{\circ}$ produtor mundial de madeira bruta em 1992, participou com menos de $2 \%$ das exportações mundiais de produtos de base florestal de maior valor agregado, como madeira serrada, painéis, pasta de madeira e papéis, denotando a potencialidade de crescimento que se apresenta para o país neste segmento (Macedo \& Mattos, 1995).

No entanto, para que as empresas do setor florestal brasileiro vislumbrem essas promissoras perspectivas de médio e longo prazo para o mercado mundial de produtos florestais, é imprescindível um planejamento criterioso da produção, ou seja, é necessário a adoção de regimes de manejo adequados para cada espécie, em cada sítio, que visem prioritariamente a qualidade do produto final. Isso quer dizer que o Brasil só poderá competir no mercado internacional de toras, por exemplo, se elas tiverem um mínimo de qualidade exigida pelo mercado.

Nesse contexto, é de grande importância a utilização de sistemas de predição do crescimento e da produção. Dentre as possibilidades para modelar o crescimento e a produção dos povoamentos florestais podem-se destacar os modelos descritivos ou biométricos e os modelos com base em processos. As duas linhas de abordagem são complementares, e não conflitivas (Kimmins et al., 1999). Enquanto os modelos por processo ou mecanicistas têm maior capacidade generalista, possuindo a tendência intrínseca de grande potência de inferência, mesmo para situações não pontualmente amostradas, os modelos descritivos reproduzem muito bem as situações do mundo real, mas estritamente dentro da base de dados considerada na sua formulação (Burkhart, 1999). Estes modelos podem ser classificados em modelos para o povoamento, modelos por classe diamétrica e modelos para árvores individuais. Assim, utilizando modelos com simulador de desbaste, é possível que vários cenários sejam traçados, simulando a implantação de regimes de manejo em terras da própria empresa ou em terras arrendadas, em áreas planas ou acidentadas e a diferentes taxas de desconto, ampliando-se o horizonte de opções na tomada de decisão de qual é o melhor regime de manejo para cada espécie, em cada sítio e para um determinado produto final (Scolforo, 1999).

Os objetivos deste trabalho foram desenvolver um sistema para prognose do crescimento e da produção de Pinus taeda L. para simular e avaliar economicamente diferentes regimes de manejo, visando produzir madeira livre de nós (clearwood) e madeira para múltiplos usos; e analisar a lucratividade dos regimes de manejo em diversas condições de sítio, espaçamento, taxas de desconto e preço da madeira, considerando plantios realizados em terras da própria empresa e em terras arrendadas.

\section{MATERIAL E MÉTODOS}

\subsection{Localização e Características da Área}

A área em estudo está situada no município de Jaguariaíva, Estado do Paraná, entre os paralelos $24^{\circ} \mathrm{e}$ $24^{\circ} 30^{\prime}$ de latitude sul e os meridianos 49 $30^{\prime}$ e $50^{\circ}$ de longitude oeste de Greenwich, com altitude variando entre 700 e $1.100 \mathrm{~m}$. 


\subsection{Modelo de Prognose do Crescimento e da Produção}

Para obtenção da prognose do crescimento e da produção do volume por classe diamétrica, para povoamentos de Pinus taeda, foi utilizado o software SPPpinus (Scolforo, 1999a), desenvolvido a partir de 432 parcelas permanentes de $500 \mathrm{~m}^{2}$ de área, sujeitas a medições anuais e nos períodos que antecederam os desbastes. Foram utilizadas desde parcelas com duas medições até outras com sete medições, devendo ser ressaltado que a amplitude de idade dessas medições variou de 4 a 28 anos.

\subsubsection{Equação para Classificação de Sítio e para os Atributos do Povoamento}

Selecionou-se o modelo que melhor representou a relação entre a altura média das árvores dominantes (Hd) e a idade (I), analisando-se o comportamento anamórfico ou polimórfico das curvas de sítio e a estabilidade destas para a base de dados em questão.

Para estimar os atributos do povoamento foram selecionados ou desenvolvidos, através do método "Stepwise", modelos para sobrevivência (N), área basal (G), diâmetro mínimo (Dmin), diâmetro máximo (Dmax) e variância dos diâmetros $\left(S^{2} d\right)$, e especificamente para estimativa da variância dos diâmetros também foi desenvolvido um modelo para estimar a média aritmética dos diâmetros $(\overline{\mathrm{D}})$. As equações resultantes estimam estes atributos por diferentes classes de número de árvores no povoamento remanescente, ou seja: estratos 1, 2, 3 e 4, as quais se referem ao número de árvores por hectare $\geq 1.400$; de 650 a 1.399; de 300 a 649; e < 300, respectivamente.

\subsubsection{Ajuste da Distribuição Diamétrica}

Para cada parcela e em cada época de medição foi comparada a freqüência acumulativa, estimada pela distribuição testada com a freqüência acumulativa observada nas parcelas. $O$ ponto de maior divergência entre as duas distribuições é o valor D de Kolmogorov-Smirnov (Sokal \& Rohlf, 1981).

A distribuição utilizada foi a Weibull, com ajuste pelo método dos momentos, cuja função de densidade de probabilidade (fdp) é

$$
f(x)=\left(\frac{c}{b}\right)\left(\frac{x-a}{b}\right)^{c-1} \exp \left[-\left(\frac{x-a}{b}\right)^{c}\right]
$$

em que $\mathrm{a} \leq \mathrm{x}<\infty, \mathrm{a} \geq 0, \mathrm{~b}>0$ e $\mathrm{c}>0$, sendo: $\mathrm{a}=$ parâmetro de locação; $b=$ parâmetro de escala; $c=$ parâmetro de forma; e x = variável de interesse, no caso diâmetro.

O parâmetro "a" foi vinculado ao diâmetro mínimo, que foi estimado através do modelo:

$D \min _{2}=\beta_{\mathrm{o}}+\beta_{1} \overline{\mathrm{D}}_{2}+\beta_{2} \mathrm{Hd}_{2}+\beta_{3} \sqrt{\mathrm{S}^{2} \mathrm{~d}_{2}+} \beta_{4} \ln \mathrm{G}_{2}+\beta_{5} \mathrm{I}_{2}$ em que Dmin, $(\bar{D}), G, S^{2}$, I e Hd já foram definidos anteriormente; o índice 1 significa presente e o índice 2 futuro ou a idade em que será realizada a prognose para aquela variável; $\ln =$ logaritmo natural; e $\beta_{\text {is }}=$ parâmetros a serem estimados.

O sistema de prognose do crescimento e da produção com simulador de desbaste desenvolvido apresenta compatibilidade entre a área basal estimada para o povoamento e pela distribuição Weibull. Esta compatibilidade se dá através do recálculo dos parâmetros "b" e "c", até que a área basal obtida a partir da distribuição Weibull seja igual à área basal do povoamento estimada pelo modelo listado a seguir, para o estrato 1 .

$$
\begin{aligned}
\ln \left(G_{2}\right) & =\left(\frac{I_{1}}{I_{2}}\right) \ln \left(G_{1}\right)+\alpha_{1}\left(1-\frac{I_{1}}{I_{2}}\right) N_{2} \\
& +\alpha_{2}\left(1-\frac{I_{1}}{I_{2}}\right) \ln \left(H_{2}\right)+\alpha_{3}\left(1-\frac{I_{1}}{I_{2}}\right)\left(\frac{1}{I_{2}^{4}}\right)
\end{aligned}
$$

O parâmetro "c" foi estimado a partir da média aritmética $(\overline{\mathrm{D}})$, do desvio-padrão dos diâmetros $(\mathrm{Sd}) \mathrm{e}$ da função gama $(\Gamma)$. O parâmetro "b" foi estimado a partir da média aritmética dos diâmetros, da função gama e do parâmetro estimado "c". Assim, como $\mathrm{Dg}^{2}-\overline{\mathrm{D}}^{2}=\mathrm{S}^{2} \mathrm{~d}$, então $\bar{D}=\sqrt{\mathrm{Dg}^{2}-\mathrm{S}^{2} \mathrm{~d}}$, sendo o diâmetro médio quadrático (Dg) expresso como $\mathrm{Dg}=\sqrt{\frac{\mathrm{G}_{2}}{0,0000785398163 \mathrm{~N}_{2}}}$ e a variância dos diâmetros estimada a partir do modelo: $\ln \mathrm{S}^{2} \mathrm{~d}=\beta_{0}+\beta_{1}(\mathrm{Dg}-\overline{\mathrm{D}})+\beta_{2} \overline{\mathrm{D}}+\beta_{3} \mathrm{Hd}^{2}+\beta_{4} \frac{1}{\mathrm{I}_{2}}+\beta_{5} \mathrm{Dg}$

de tal maneira que a média aritmética dos diâmetros para recálculo dos parâmetros foi obtida como:

$$
\overline{\mathrm{D}}=\sqrt{\left(\frac{\mathrm{G}_{2}}{0,0000785398163 \mathrm{~N}_{2}}\right)-\exp \left[\ln \left(\mathrm{S}^{2} \mathrm{~d}\right)\right]}
$$

R. Árvore, Viçosa-M G, v.26, n.6, p.699-713, 2002 
Assim, conhecida a estimativa da média aritmética e do desvio-padrão dos diâmetros, obtêm-se o coeficiente de variação (CV) e, então, o parâmetro "c" é estimado a partir de técnica iterativa, através da expressão:

$$
\mathrm{CV}=\frac{\mathrm{Sd}}{\overline{\mathrm{D}}}=\frac{\left[\Gamma(1+2 / \mathrm{c})-\Gamma^{2}(1+1 / \mathrm{c})\right]^{1 / 2}}{\Gamma(1+1 / \mathrm{c})}
$$

Conhecido "c", o parâmetro "b" pode ser estimado como:

$\mathrm{b}=\frac{\overline{\mathrm{D}}}{\Gamma(1+1 / \mathrm{c})}$. Assim, os parâmetros "b" e “c" são recalculados até que a soma da área basal obtida da distribuição Weibull (1) seja similar à área basal (G) estimada pela equação do povoamento (3).

\subsubsection{Função de Afilamento}

O modelo adotado foi construído a partir de um Stepwise, no qual foram utilizadas as potências: 0,005 ; 0,$09 ; 0,08 ; 0,07 ; 0,06 ; 0,05 ; 0,04 ; 0,03 ; 0,02 ; 0,01 ; 0,9$; 0,$8 ; 0,7 ; 0,6 ; 0,5 ; 0,4 ; 0,3 ; 0,2 ; 0,1 ; 1 ; 2 ; 3 ; 4 ; 5 ; 10 ; 15$; 20; e 25, associadas ao polinômio:

$$
\frac{\mathrm{d}_{\mathrm{i}}}{\mathrm{D}}=\beta_{0}+\beta_{1}\left(\frac{\mathrm{h}_{\mathrm{i}}}{\mathrm{H}}\right)^{\mathrm{p} 1}+\beta_{2}\left(\frac{\mathrm{h}_{\mathrm{i}}}{\mathrm{H}}\right)^{\mathrm{p} 2}+\ldots+\beta_{\mathrm{n}}\left(\frac{\mathrm{h}_{\mathrm{i}}}{\mathrm{H}}\right)^{\mathrm{pn}}+\varepsilon_{\mathrm{i}}
$$

em que $\mathrm{d}_{\mathrm{i}}=$ diâmetro comercial $(\mathrm{cm}) ; \mathrm{D}=$ diâmetro à altura do peito $(\mathrm{cm}) ; \mathrm{H}=$ altura total $(\mathrm{m}) ; \mathrm{h}_{\mathrm{i}}=$ altura comercial $(\mathrm{m}) ; \beta_{\text {is }}=$ parâmetros a serem estimados; $\varepsilon_{\mathrm{i}}=$ erro de estimativa; e $\mathrm{p}_{\mathrm{i}}=$ expoentes variando entre 0,005 e 25 .

A base técnica adotada para a amplitude de potência utilizada foi que potências com grandezas de dezenas possibilitam melhor ajuste do polinômio para a base da árvore, potências com grandezas unitárias possibilitam melhor ajuste para a porção intermediária da árvore e potências fracionárias possibilitam melhor ajuste para a ponta da árvore, conforme estudos de Hradetzky (1976), Rosot (1989), Fischer (1997), Fischer et al. (2001) e Scolforo (1999b).

\subsubsection{Modelo de Desbaste}

O modelo definido para retratar os diferentes números, épocas e intensidades de desbastes a serem aplicados ao povoamento foi

$$
P_{i}=\exp \left[\beta_{1}\left(\frac{d_{i}^{2}}{d^{2}}\right)^{\beta_{2}}\right]
$$

em que $\mathrm{P}_{\mathrm{i}}=$ proporção de área basal removida na classe de diâmetro i; $\mathrm{D}_{\mathrm{i}}=$ centro da classe de diâmetro i; $\mathrm{Dg}=$ diâmetro médio quadrático do povoamento; e $\beta_{1}, \beta_{2}=$ parâmetros a serem estimados.

\subsubsection{Estimativa da Produção}

Foram simuladas diversas alternativas de regimes de manejo, enquadradas em dois cenários, com diferentes números, épocas e intensidade de desbaste, a partir de várias densidades iniciais de plantio, em diferentes sítios. Para obtenção de madeira livre de nós, foi considerado que o primeiro desbaste é pré-comercial, realizado em idades jovens, seguido de desrama.

A primeira desrama ocorre simultaneamente ao desbaste pré-comercial, e as árvores selecionadas são desramadas até a altura mínima de 2,4 m, desde que não ultrapasse $50 \%$ da altura da árvore ou um terço de sua copa viva. A segunda desrama ocorre em torno dos 7 e 8 anos, atingindo a altura de 6,7 a 7,0 m nas árvores selecionadas.

Para implementar o sistema, foram adotados:

a) Regimes de manejo a partir de três densidades iniciais de plantio em três sítios, conforme pode ser visto no Quadro 1.

b) Cenários propriamente ditos: foram estabelecidos com base nas nove opções definidas no Quadro 1, variando o número, a época de ocorrência dos desbastes pré-comerciais e comerciais e do corte final, assim como a intensidade dos desbastes.

b.1.) Cenário 1: serão realizados um desbaste précomercial e um desbaste comercial em diferentes épocas, diversas intensidades de remoção e diferentes idades de corte final. No Quadro 2 estão listadas estas opções, considerando a densidade inicial de plantio de 1.333 árvores/ ha, no índice de sítio 28,5 m. Para as outras oito situações definidas no Quadro 1, a mesma sistemática foi adotada.

Na idade de 4 anos, quando é realizado o desbaste pré-comercial, é possível deixar 500, 550 ou 600 árvores remanescentes, e o desbaste comercial pode ser realizado aos 8, 10 ou 12 anos, deixando 200, 250 ou 300 árvores remanescentes e executando o corte final aos 20,21 ou 22 anos, sendo possível realizar 81 diferentes alternativas de regimes de manejo. Multiplicando esse valor por 3, uma vez que o desbaste pré-comercial pode ocorrer aos 4, 5 ou 6 anos, chega-se ao número de 243 possíveis 
combinações. Portanto, para cada uma das nove situações do Quadro 1 existem 243 possíveis combinações, resultando em um número total de 2.187 alternativas de regimes de manejo para o cenário 1 .

Quadro 1 - Densidades iniciais de plantio e sítios considerados no teste das opções de regimes de manejo Table 1 - Initial densities in various sites

\begin{tabular}{|c|c|c|}
\hline Sítio & $\begin{array}{c}\text { Espaçamento } \\
(\mathrm{m} \times \mathrm{m})\end{array}$ & Densidade Inicial de Plantio \\
\hline I & $3,0 \times 2,5$ & 1.333 árvores $/ \mathrm{ha}$ \\
$(\mathrm{S}=28,5 \mathrm{~m})$ & $3,0 \times 3,0$ & 1.111 árvores $/ \mathrm{ha}$ \\
& $3,0 \times 4,0$ & 833 árvores $/ \mathrm{ha}$ \\
\hline \multirow{2}{*}{ II } & $3,0 \times 2,5$ & 1.333 árvores $/ \mathrm{ha}$ \\
$(\mathrm{S}=25,5 \mathrm{~m})$ & $3,0 \times 3,0$ & 1.111 árvores $/ \mathrm{ha}$ \\
& $3,0 \times 4,0$ & 833 árvores $/ \mathrm{ha}$ \\
\hline III & $3,0 \times 2,5$ & 1.333 árvores $/ \mathrm{ha}$ \\
$(\mathrm{S}=22,5 \mathrm{~m})$ & $3,0 \times 3,0$ & 1.111 árvores $/ \mathrm{ha}$ \\
& $3,0 \times 4,0$ & 833 árvores $/ \mathrm{ha}$ \\
\hline
\end{tabular}

$\mathrm{S}$ - índice de sítio na idade de referência de 20 anos.

b.2.) Cenário 2: serão realizados um desbaste précomercial e dois desbastes comerciais em diferentes épocas, com diversas intensidades de remoção e idades de corte final. No Quadro 3 estão listadas estas opções, considerando a densidade inicial de plantio de 1.333 árvores/ha, no índice de sítio 28,5 m. Para as outras oito situações definidas no Quadro 1, a mesma sistemática foi adotada.

\section{c) Geração dos Regimes de Manejo}

Usando o sistema SPPpinus foram simulados os regimes de manejo propostos no item (b). Os sortimentos ou múltiplos produtos considerados estão no Quadro 4.

\subsection{Análise Econômica}

\subsubsection{Estrutura de Custos e Preços}

O Quadro 5 mostra os custos do plantio, os custos de tratos silviculturais e os custos de proteção florestal, todos expressos em $\mathrm{R} \$$ por hectare. Como estes dois últimos são terceirizados, eles são computados até o terceiro ano. Incide sobre os três custos anteriores uma taxa de administração de $10 \%$. De forma complementar, durante toda a vida do povoamento ocorre também o custo de administração direta ou overhead.

No Quadro 6 estão os custos de colheita (corte, arraste e carregamento) dos desbastes e do corte final, todos expressos em $\mathrm{R} \$ / \mathrm{m}^{3}$. Eles ocorrem no ano de corte, sendo diferenciados de acordo com o volume médio das árvores retiradas em cada intervenção.

No Quadro 7 encontram-se os preços de venda dos diferentes produtos, para os quais se obteve estimativa volumétrica.

\subsubsection{Método para Avaliação Econômica dos Regimes de Manejo}

Foram utilizados dois critérios de análise econômica, considerando o valor presente líquido de uma série

Quadro 2 - Idades dos desbastes e do corte final e número de árvores remanescentes para a densidade inicial de 1.333 árvores/ha, no índice de sítio $28,5 \mathrm{~m}$, para dois desbastes

Table 2 -Thinning and final cutting ages and number of remaining trees, for an initial density of 1,333 trees per hectare, in the site index of $28,5 \mathrm{~m}$, for two thinnings

\begin{tabular}{|c|c|c|c|c|c|c|c|c|}
\hline $\begin{array}{c}\text { Idade do Desbaste Pré- } \\
\text { comercial } \\
\text { (anos) }\end{array}$ & & $\begin{array}{l}\text { o de } \\
\text { lanesc } \\
\left(\mathrm{ha}^{-1}\right)\end{array}$ & & $\begin{array}{l}\text { Idade do } 1^{\circ} \text { Des- } \\
\text { Baste Comercial } \\
\quad(\text { anos })\end{array}$ & $\begin{array}{c}\text { Número de Árvores } \\
\text { Remanescentes } \\
\left(\mathrm{ha}^{-1}\right)\end{array}$ & & ano & \\
\hline \multirow{9}{*}{4,5 ou 6} & \multirow{9}{*}{500} & \multirow{9}{*}{550} & \multirow{9}{*}{600} & \multirow{3}{*}{8} & 200 & 20 & 21 & 22 \\
\hline & & & & & 250 & 20 & 21 & 22 \\
\hline & & & & & 300 & 20 & 21 & 22 \\
\hline & & & & \multirow{3}{*}{10} & 200 & 20 & 21 & 22 \\
\hline & & & & & 250 & 20 & 21 & 22 \\
\hline & & & & & 300 & 20 & 21 & 22 \\
\hline & & & & \multirow{3}{*}{12} & 200 & 20 & 21 & 22 \\
\hline & & & & & 250 & 20 & 21 & 22 \\
\hline & & & & & 300 & 20 & 21 & 22 \\
\hline
\end{tabular}


infinita de rotações. Estes se diferenciam pela inclusão ou não do custo da terra.

\section{a) Valor Presente Líquido Geral (VPG)}

O "VPG" considera o custo de oportunidade pelo uso da terra, ou seja, a terra pode ser mantida para o presente povoamento, convertida em outro povoamento ou vendida para outros usos. Este critério pode ser expresso da seguinte forma:

$$
V P G=\frac{\sum_{n=0}^{t}\left(R_{n}-C_{n}\right) \cdot(1+t)^{t-n}-\left[L \cdot(1+i)^{t}\right]}{\left((1+i)^{t}-1\right)}
$$

em que $\mathrm{L}=$ custo da terra; $\mathrm{t}=$ idade de rotação; $\mathrm{R}_{\mathrm{n}}=$ receitas no ano $n ; C_{n}=$ custos no ano $n ;$ e $i=$ taxa de juros comparativa ou taxa mínima de atratividade.

Bentley \& Teenguarden (1965) e Gomes (1999) destacam que o VPG concede para a terra somente o seu preço de mercado presente e que algum excedente é tratado como uma renda capturada pelo proprietário, acima da qual ele desejaria para remunerar seu salário e capital.

\section{b) Valor Esperado da Terra (VET)}

A pressuposição peculiar do modelo de renda do solo ou valor esperado da terra é que não existe acesso aos mercados de terra, isto é, a terra é um fator de produção fixo e, conseqüentemente, todos os excedentes econômicos resultam para a terra (Bentley \& Teenguarden, 1965). Estabelecendo-se o valor presente líquido geral (VPG) igual a zero, o VET pode ser obtido como se segue:

$$
\operatorname{VET}=\frac{\sum_{n=0}^{t}\left(R_{n}-C_{n}\right) \cdot(1+i)^{t-n}}{\left((1+i)^{t}-1\right)}
$$

Para calcular o VET utilizou-se o software Invest (Scolforo, 1999b), que é integrado ao software SPPpinus, fato este que permite simular variações nas taxas de desconto, nas distâncias de transporte, nos valores de custos e de preços dos produtos e na declividade da área explorada (acidentada ou plana), como também analisar regimes de manejo conduzidos em terras próprias ou em terras arrendadas.

\subsubsection{Análise de Sensibilidade}

Analisou-se a sensibilidade do VET dos diversos regimes de manejo a taxas de juros de 6, 8 e 10\% a.a., considerando terras da própria empresa ou arrendadas e uma variação do preço de venda da madeira desramada em relação à madeira não-desramada. Foram analisadas

Quadro 3 - Idades dos desbastes e do corte final e número de árvores remanescentes para uma densidade inicial de 1.333 árvores/ha, no índice de sítio $28,5 \mathrm{~m}$, para três desbastes

Table 3 - Thinning and final cutting ages and number of remaining trees, for initial density of 1,333 trees per hectare, in

\begin{tabular}{|c|c|c|c|c|c|c|c|c|c|}
\hline $\begin{array}{c}\text { Idade do } \\
\text { Desbaste Pré- } \\
\text { comercial } \\
\text { (anos) }\end{array}$ & $\begin{array}{r}\text { Nú } \\
\text { Á } \\
\text { Rema }\end{array}$ & $\begin{array}{l}\text { o de } \\
\text { es } \\
\text { centes } \\
\text { ) }\end{array}$ & $\begin{array}{c}\text { Idade do } 1^{\mathrm{o}} \\
\text { Desbaste } \\
\text { Comercial } \\
\text { (anos) }\end{array}$ & $\begin{array}{c}\text { Número de } \\
\text { Árvores } \\
\text { Remanescentes } \\
\left(\mathrm{ha}^{-1}\right)\end{array}$ & $\begin{array}{c}\text { Idade do } 2^{\underline{0}} \\
\text { Desbaste } \\
\text { Comercial } \\
\text { (anos) }\end{array}$ & $\begin{array}{r}\text { Nú } \\
\text { Á } \\
\text { Reme }\end{array}$ & $\begin{array}{l}\text { de } \\
\text { es } \\
\text { centes }\end{array}$ & $\begin{array}{l}\mathrm{Id} \\
\mathrm{Co}\end{array}$ & $\begin{array}{l}\text { de } \\
\text { inal } \\
\text { ) }\end{array}$ \\
\hline \multirow{12}{*}{4,5 ou 6} & \multirow{12}{*}{500} & \multirow{12}{*}{600} & \multirow{6}{*}{10} & \multirow{3}{*}{300} & 14 & 100 & 200 & 20 & 21 \\
\hline & & & & & 15 & 100 & 200 & 20 & 21 \\
\hline & & & & & 16 & 100 & 200 & 20 & 21 \\
\hline & & & & \multirow{3}{*}{400} & 14 & 100 & 200 & 20 & 21 \\
\hline & & & & & 15 & 100 & 200 & 20 & 21 \\
\hline & & & & & 16 & 100 & 200 & 20 & 21 \\
\hline & & & \multirow{6}{*}{12} & \multirow{3}{*}{300} & 14 & 100 & 200 & 20 & 21 \\
\hline & & & & & 15 & 100 & 200 & 20 & 21 \\
\hline & & & & & 16 & 100 & 200 & 20 & 21 \\
\hline & & & & \multirow{3}{*}{400} & 14 & 100 & 200 & 20 & 21 \\
\hline & & & & & 15 & 100 & 200 & 20 & 21 \\
\hline & & & & & 16 & 100 & 200 & 20 & 21 \\
\hline
\end{tabular}
the site index of $28.5 \mathrm{~m}$, for three thinnings 
duas situações: preço da madeira desramada igual ao preço da madeira não-desramada e preço da madeira desramada $40 \%$ superior ao preço da madeira não-desramada. Estas simulações geraram 26.244 e 31.104 regimes de manejo para os cenários 1 e 2 , respectivamente.

Quadro 4 - Produtos e suas especificações de acordo com cada uso da madeira

Table 4 - Products and their specifications, according to each wood use

\begin{tabular}{|l|c|c|c|}
\hline \multirow{2}{*}{\multicolumn{1}{|c|}{ Produto }} & \multicolumn{3}{|c|}{ Dimensão } \\
\cline { 2 - 3 } & \multicolumn{2}{|c|}{ Diâmetro de uso } & Comprimento \\
\cline { 2 - 3 } & $\begin{array}{c}\text { Mínimo } \\
(\mathrm{cm})\end{array}$ & $\begin{array}{c}\text { Máximo } \\
(\mathrm{cm})\end{array}$ & $(\mathrm{m})$ \\
\hline Madeira para processo & 8 & 17,9 & 2,2 \\
Madeira para serraria & 18 & 24,9 & 2,2 \\
Madeira para laminação & 25 & 34,9 & 2,2 \\
Madeira especial & $\geq 35$ & & 2,2 \\
\hline
\end{tabular}

Quadro 5 - Custos de manejo, em $\mathrm{R} \$ /$ ha

Table 5 - Management costs per hectare $(R \$ / h a)$

\begin{tabular}{|l|c|c|}
\hline \multicolumn{1}{|c|}{ Operação } & $\begin{array}{c}\text { Ano de } \\
\text { Ocorrência }\end{array}$ & $\begin{array}{c}\text { Custos } \\
\text { (R\$/ha) }\end{array}$ \\
\hline 1. Infra-estrutura (estradas, aceiros, ...) & 0 & 42,00 \\
2. Preparo do terreno & 0 & 201,82 \\
3. Implantação de florestas & 0 & 218,04 \\
\hline Total até o plantio & & 461,86 \\
\hline 4. Tratos culturais & 1 & 119,42 \\
5. Tratos culturais & 2 & 95,15 \\
6. Tratos culturais & 3 & 38,54 \\
7. Proteção florestal & 1 & 28,24 \\
8. Proteção florestal & 2 & 28,24 \\
9. Proteção florestal & 3 & 28,24 \\
10. Administração & 1 a n* & 80,08 \\
11. Overhead & 1 a n* & 25,00 \\
12. Preço da terra para a classe de sítio & & 1500,00 \\
I ou índice de sítio (S) 28,5 m & & \\
13. Preço da terra para a classe de sítio & & 1200,00 \\
II ou índice de sítio (S) 25,5 m & & 900,00 \\
14. Preço da terra para a classe de sítio & & Igual a $20 \%$ \\
III ou índice de sítio (S) 22,5 m & & bruta \\
\hline 15. Arrendamento de terra & & \\
& &
\end{tabular}

$\mathrm{n}^{*}=$ idade do corte final.
Quadro 6 - Custos de colheita $\left(\mathrm{R} \$ / \mathrm{m}^{3}\right)$ por classe de uso e de volume

Table 6 - Logging costs in $\left(R \$ / m^{3}\right)$ per use and volume class

\begin{tabular}{|c|c|c|c|c|}
\hline \multirow{2}{*}{$\begin{array}{c}\text { Classe de } \\
\text { Volume }\end{array}$} & \multicolumn{4}{|c|}{ Classe de Uso } \\
\cline { 2 - 5 } & $\begin{array}{c}\text { Processo } \\
(8 \vdash 18)\end{array}$ & $\begin{array}{c}\text { Serraria } \\
(18 \vdash 25)\end{array}$ & $\begin{array}{c}\text { Laminação } \\
(25 \vdash 35)\end{array}$ & $\begin{array}{c}\text { Madeira } \\
\text { Especial } \\
(\geq 35)\end{array}$ \\
\hline $0,10 \vdash 0,20$ & 8,523 & 8,631 & 8,708 & 8,708 \\
$0,20 \vdash 0,33$ & 7,154 & 7,261 & 7,338 & 7,338 \\
$0,33 \vdash 0,46$ & 6,923 & 7,031 & 7,108 & 7,108 \\
$0,46 \vdash 0,59$ & 7,154 & 7,261 & 7,338 & 7,338 \\
$\geq 0,59$ & 7,415 & 7,523 & 7,600 & 7,600 \\
\hline
\end{tabular}

$\mathrm{O}$ custo da desrama varia de acordo com o número de árvores a serem desramadas por hectare e também de acordo com a altura da desrama.

Quadro 7 - Preços de venda dos diversos produtos obtidos da madeira não-desramada

Table 7 - Products, gauges and market prices

\begin{tabular}{|l|c|c|}
\hline \multicolumn{1}{|c|}{ Produto } & Bitola $(\mathrm{cm})$ & $\begin{array}{c}\text { Preço de Venda } \\
\left(\mathrm{R} \$ / \mathrm{m}^{3}\right)\end{array}$ \\
\hline Processo & $8 \vdash 18$ & 10,32 \\
Serraria & $18 \vdash 25$ & 17,70 \\
Laminação & $25 \vdash 35$ & 24,60 \\
Madeira especial & $\geq 35$ & 44,30 \\
\hline
\end{tabular}

\section{RESULTADOS E DISCUSSÕES}

\subsection{O Modelo para Prognose do Crescimento e da Produção}

No Quadro 8 estão as equações para a estimativa do índice de sítio e a relação entre a média aritmética em altura das árvores dominantes com a idade, assim como suas medidas de precisão.

A relação altura média das árvores dominantes com a idade apresentou forma polimórfica. O teste de estabilidade aplicado mostrou que $69 \%$ das parcelas, em suas diversas medições, não apresentaram qualquer alteração no seu índice de sítio, 11,8\% foram parcelas que se situaram nas diversas medições no limite superior de um sítio e inferior de outro e as demais apresentaram uma mudança no valor de índice de sítio, fato verificado principalmente na primeira idade de medição das parcelas, quando avaliadas aos 5 anos. Mesmo para estas parcelas pode-se observar total estabilidade nos seus valores de índice de sítio, nas demais idades de medição. 
As estatísticas obtidas para esse componente fundamental do modelo de crescimento e produção são indicadoras da alta qualidade que se obteve em sua construção.

No Quadro 9 estão as equações para a estimativa dos atributos do povoamento por classe de número de árvores remanescentes, assim com suas medidas de precisão.

A equação para a estimativa da média aritmética $(\mathrm{H})$ em cada classe diamétrica $\left(\mathrm{D}_{\mathrm{i}}\right)$, em função do tempo, da densidade do povoamento (Dg) e de uma medida de produtividade do local (Hd), é

$$
\begin{aligned}
H\left(D_{i}\right) & =\exp \left[0,224903+0,924565 \ln \mathrm{Hd}_{2}-2,287734 \frac{1}{\mathrm{D}_{\mathrm{i}}}\right. \\
& \left.-0,258581 \ln \left(\frac{\mathrm{Dg}_{2}}{\mathrm{D}_{\mathrm{i}}}\right)+0,141775\left(\frac{1}{\mathrm{I}_{2} \mathrm{D}_{\mathrm{i}}}\right)\right]
\end{aligned}
$$

com coeficiente de determinação $\left(\mathrm{R}^{2}\right)$ de $96,7 \%$ e erropadrão dos resíduos $\left(\mathrm{S}_{\mathrm{YX}}\right)$ de $\pm 0,9058 \mathrm{~m}$.

A equação de afilamento desenvolvida por Stepwise foi com potência fracionária e inteira, como:

$$
\begin{aligned}
\mathrm{d}_{\mathrm{i}}=\mathrm{D}\left[1,2606+0,26445\left(\frac{\mathrm{h}_{\mathrm{i}}}{\mathrm{H}}\right)^{0,00001}-1,04086\left(\frac{\mathrm{h}_{\mathrm{i}}}{\mathrm{H}}\right)^{0,2}\right. \\
\left.+0,66834\left(\frac{\mathrm{h}_{\mathrm{i}}}{\mathrm{H}}\right)-1,02726\left(\frac{\mathrm{h}_{\mathrm{i}}}{\mathrm{H}}\right)^{2}-0,12574\left(\frac{\mathrm{h}_{\mathrm{i}}}{\mathrm{H}}\right)^{5}\right]
\end{aligned}
$$

com coeficiente de determinação de $98,63 \%$ e erro-padrão dos resíduos de 5,66\%. O perfil médio representado por esta equação é mostrado na Figura 1 para a média das árvores cubadas rigorosamente. Pode-se constatar que não há qualquer tendenciosidade nas estimativas do diâmetro e do volume.

No Quadro 10 estão apresentadas, para fins de exemplificação, algumas possibilidades desenvolvidas no SPPpinus para descrever diferentes épocas, números e intensidade de desbastes.

\subsection{Análise Econômica dos Regimes de Manejo Gerados para o Cenário 1}

Ao analisar os 26.244 regimes de manejo gerados para o cenário 1, verificou-se que os lucros gerados pelo investimento em índices de sítio de alta produtividade $(\mathrm{S}=28,5 \mathrm{~m})$ situam-se na faixa de $\mathrm{R} \$ 1.400,00$ a $\mathrm{R} \$ 9.400,00$ por hectare. Na classe de sítio II o lucro está na faixa de $\mathrm{R} \$ 1,00$ a $\mathrm{R} \$ 6.900,00$ por hectare, e na classe de sítio III ocorreram situações em que os regimes de manejo foram inviáveis economicamente, quando o preço da madeira desramada foi igual ao preço da madeira nãodesramada. Contudo, ao considerar o acréscimo de $40 \%$ no preço da madeira desramada, em relação ao da nãodesramada, todos os regimes de manejo desse sítio tornaram-se viáveis economicamente, obtendo-se lucros de até $\mathrm{R} \$ 5.100,00$ por hectare. Tal fato indica que nem sempre o aumento de custos implica redução da rentabilidade do investimento, principalmente se este aumento de custo estiver associado à implementação de práticas de manejo que visem melhorar a qualidade do produto final e, conseqüentemente, o seu valor no mercado.

Quadro 8 - Equações para predizer o índice de sítio e a altura dominante-idade, na idade de referência 20 anos Table 8-Equations to predict site index and dominant tree average height

\begin{tabular}{|l|c|c|c|}
\hline Atributo & Equações & $\mathrm{R}^{2}(\%)$ & Syx (m) \\
\hline Índice de sítio (S) & $\mathrm{S}=\exp (5,75103856)\left[\frac{\mathrm{Hd}}{\exp (5,75103856)}\right]\left(\frac{\mathrm{I}}{\mathrm{Iref}}\right)^{0,23068992}$ & 98,1 & $\pm 1,00$ \\
$\mathrm{Hd}$ & $\mathrm{Hd}_{2}=\exp (5,75103856)\left[\frac{\mathrm{Hd}_{1}}{\exp (5,75103856)}\right]\left(\frac{\mathrm{I}_{1}}{\mathrm{I}_{2}}\right)^{0,23068992}$ & \\
\hline
\end{tabular}


Quadro 9 - Equações para predizer os atributos do povoamento Table 9 - Equations to predict stand features

\begin{tabular}{|c|c|c|c|c|}
\hline \multirow[t]{2}{*}{ Atributo } & \multirow{2}{*}{$\begin{array}{l}\text { Número de } \\
\text { Árvores }\end{array}$} & \multirow[t]{2}{*}{ Equação } & \multicolumn{2}{|c|}{$\begin{array}{l}\text { Medida de } \\
\text { Precisão }\end{array}$} \\
\hline & & & $\mathrm{R}^{2}$ & Syx \\
\hline $\begin{array}{l}\text { Sobrevi- } \\
\text { vência (N) }\end{array}$ & \begin{tabular}{|c|}
$\geq 1.400$ \\
$1.399-650$ \\
$649-300$ \\
$<300$ \\
\end{tabular} & $8 S)]\}$ & & \\
\hline $\begin{array}{l}\text { Área basal } \\
(\mathrm{G}) \\
\left(\mathrm{m}^{2} / \mathrm{ha}\right)\end{array}$ & $\begin{array}{l}1.399-650 \\
649-300\end{array}$ & $\begin{array}{l}\mathrm{G}_{2}=\left(\frac{\mathrm{I}_{1}}{\mathrm{I}_{2}}\right) \mathrm{G}_{1}+0,00691\left(1-\frac{\mathrm{I}_{1}}{\mathrm{I}_{2}}\right) \mathrm{N}_{2}+22,10179\left(1-\frac{\mathrm{I}_{1}}{\mathrm{I}_{2}}\right) \ln \mathrm{Hd}_{2}-5487,9553\left(1-\frac{\mathrm{I}_{1}}{\mathrm{I}_{2}}\right) \frac{1}{\mathrm{I}_{2}^{4}} \\
\mathrm{G}_{2}=\left(\frac{\mathrm{I}_{1}}{\mathrm{I}_{2}}\right) \mathrm{G}_{1}-5,1346361\left(1-\frac{\mathrm{I}_{1}}{\mathrm{I}_{2}}\right) \ln \mathrm{N}_{2}+36,2380353\left(1-\frac{\mathrm{I}_{1}}{\mathrm{I}_{2}}\right) \ln \mathrm{Hd}_{2}-0,0224422\left(1-\frac{\mathrm{I}_{1}}{\mathrm{I}_{2}}\right) \mathrm{I}_{2}^{2} \\
\mathrm{G}_{2}=\left(\frac{\mathrm{I}_{1}}{\mathrm{I}_{2}}\right) \mathrm{G}_{1}+0,0767763\left(1-\frac{\mathrm{I}_{1}}{\mathrm{I}_{2}}\right) \mathrm{N}_{2}+3,3067771\left(1-\frac{\mathrm{I}_{1}}{\mathrm{I}_{2}}\right) \mathrm{Hd}_{2}-14,7852985\left(1-\frac{\mathrm{I}_{1}}{\mathrm{I}_{2}}\right) \ln \mathrm{I}_{2} \\
\mathrm{G}_{2}=\left(\frac{\mathrm{I}_{1}}{\mathrm{I}_{2}}\right) \mathrm{G}_{1}+0,191633\left(1-\frac{\mathrm{I}_{1}}{\mathrm{I}_{2}}\right) \mathrm{N}_{2}+5,139108\left(1-\frac{\mathrm{I}_{1}}{\mathrm{I}_{2}}\right) \mathrm{Hd}_{2}-35,866413\left(1-\frac{\mathrm{I}_{1}}{\mathrm{I}_{2}}\right) \ln \mathrm{I}_{2}\end{array}$ & $\begin{array}{c}97,8 \\
97,7 \\
99,03 \\
99,73\end{array}$ & $\begin{array}{l} \pm 1,57 \\
\pm 1,27 \\
\pm 1,03\end{array}$ \\
\hline $\begin{array}{l}\text { Diâmetro } \\
\text { máximo } \\
(\text { Dmax }) \\
(\mathrm{cm})\end{array}$ & $1.399-650$ & $\begin{array}{l}\ln \operatorname{Dmax}_{2}=3,292071+0,425769\left(\frac{\mathrm{I}_{1}}{\mathrm{I}_{2}}\right) \ln \mathrm{Hd}_{2}+0,083226\left(\frac{\mathrm{I}_{1}}{\mathrm{I}_{2}}\right) \ln \mathrm{I}_{2}-0,358872\left(\frac{\mathrm{I}_{1}}{\mathrm{I}_{2}}\right) \ln \mathrm{N}_{2}+0,350504\left(\frac{\mathrm{I}_{1}}{\mathrm{I}_{2}}\right) \ln \mathrm{G}_{2} \\
\ln \operatorname{Dmax}_{2}=3,606733+0,184437\left(\frac{\mathrm{I}_{1}}{\mathrm{I}_{2}}\right) \ln \mathrm{Hd}_{2}+0,213924\left(\frac{\mathrm{I}_{1}}{\mathrm{I}_{2}}\right) \ln \mathrm{I}_{2}-0,367812\left(\frac{\mathrm{I}_{1}}{\mathrm{I}_{2}}\right) \ln \mathrm{N}_{2}+0,354854\left(\frac{\mathrm{I}_{1}}{\mathrm{I}_{2}}\right) \ln \mathrm{G}_{2} \\
\ln \operatorname{Dmax}_{2}=3,702007+0,12761\left(\frac{\mathrm{I}_{1}}{\mathrm{I}_{2}}\right) \ln \mathrm{Hd}_{2}+0,014409\left(\frac{\mathrm{I}_{1}}{\mathrm{I}_{2}}\right) \ln \mathrm{I}_{2}-0,38848\left(\frac{\mathrm{I}_{1}}{\mathrm{I}_{2}}\right) \ln \mathrm{N}_{2}+0,55834\left(\frac{\mathrm{I}_{1}}{\mathrm{I}_{2}}\right) \ln \mathrm{l}_{2} \\
\ln \operatorname{Dmax}_{2}=3,752747+0,172636\left(\frac{\mathrm{I}_{1}}{\mathrm{I}_{2}}\right) \ln \mathrm{Hd}_{2}+0,149972\left(\frac{\mathrm{I}_{1}}{\mathrm{I}_{2}}\right) \ln \mathrm{I}_{2}-0,381721\left(\frac{\mathrm{I}_{1}}{\mathrm{I}_{2}}\right) \ln \mathrm{N}_{2}+0,351186\left(\frac{\mathrm{I}_{1}}{\mathrm{I}_{2}}\right) \ln \mathrm{G}_{2}\end{array}$ & $\begin{array}{l}90,2 \\
99,9\end{array}$ & $\begin{array}{l} \pm 0,06 \\
\pm 0,05 \\
\pm 0,001\end{array}$ \\
\hline $\begin{array}{l}\text { Média dos } \\
\text { diâmetros } \\
\overline{(\bar{D}}) \\
(\mathrm{cm})\end{array}$ & $\begin{array}{c}\geq 1.400 \\
1.399-650 \\
649-300 \\
<300\end{array}$ & $\begin{array}{l}\overline{\mathrm{D}}_{2}=1,07866+1,05685 \mathrm{Dg}_{2}-0,8264\left(\mathrm{Hd}_{2}\right)^{0,2}-0,006088 \mathrm{I}_{2}-0,04138 \mathrm{D} \max _{2} \\
\overline{\mathrm{D}}_{2}=2,894958+1,068239 \mathrm{Dg}_{2}-1,978826\left(\mathrm{Hd}_{2}\right)^{0,2}+0,020625 \mathrm{I}_{2}-0,053638 \mathrm{Dmax}_{2} \\
\overline{\mathrm{D}}_{2}=2,640205+1,045739 \mathrm{Dg}_{2}-1,729637\left(\mathrm{Hd}_{2}\right)^{0,2}+0,009433 \mathrm{I}_{2}-0,034413 \mathrm{D} \max _{2} \\
\overline{\mathrm{D}}_{2}=-82,032587+1,948516 \mathrm{Dg}_{2}+54,671026\left(\mathrm{Hd}_{2}\right)^{0,2}+0,49578 \mathrm{II}_{2}-1,498878 \mathrm{D} \max _{2}\end{array}$ & $\begin{array}{l}99,9 \\
99,8 \\
99,9 \\
99,9\end{array}$ & $\begin{array}{l} \pm 0,15 \\
\pm 0,15 \\
\pm 0,14 \\
\pm 0,01\end{array}$ \\
\hline & $\geq 1.400$ & $\mathrm{~S}^{2} \mathrm{~d}_{2}=-14,448086-16,358906 \sqrt{\mathrm{Dg}_{2}-\overline{\mathrm{D}_{2}}}-45,013415 \overline{\mathrm{D}_{2}}+0,020635 \mathrm{Hd}_{2}^{2}+33,246783\left(\frac{1}{\mathrm{I}_{2}}\right)+45,791453 \mathrm{Dg}_{2}$ & 98,0 & $\pm 2,29$ \\
\hline $\begin{array}{l}\text { Variância } \\
\text { dos } \\
\text { diâmetros } \\
\left(\mathrm{S}^{2} \mathrm{~d}\right) \\
\left(\mathrm{cm}^{2}\right)\end{array}$ & $\begin{array}{l}649-300 \\
<300\end{array}$ & $\begin{array}{l}\mathrm{S}^{2} \mathrm{~d}_{2}=-23,432177-24,427486 \sqrt{\mathrm{Dg}_{2}-\overline{\mathrm{D}_{2}}}-67,164646 \overline{\mathrm{D}}_{2}+0,01024 \mathrm{Hd}_{2}^{2}+55,596564\left(\frac{1}{\mathrm{I}_{2}}\right)+68,136228 \mathrm{Dg}_{2} \\
\mathrm{~S}^{2} \mathrm{~d}_{2}=-9,738453-53,838056 \sqrt{\mathrm{Dg}_{2}-\overline{\mathrm{D}_{2}}}-101,066283 \overline{\mathrm{D}}_{2}+0,001123 \mathrm{Hd}_{2}^{2}+1,810042\left(\frac{1}{\mathrm{I}_{2}}\right)+101,991623 \mathrm{Dg}_{2} \\
\mathrm{~S}^{2} \mathrm{~d}_{2}=-3,838950-0,248112 \sqrt{\mathrm{Dg}_{2}-\overline{\mathrm{D}_{2}}}-4,331359 \ln \overline{\mathrm{D}}_{2}-6,640293 \ln \mathrm{Hd}_{2}^{2}+0,439092 \ln \mathrm{I}_{2}\end{array}$ & $\begin{array}{l}97,7 \\
99,9\end{array}$ & $\begin{array}{l} \pm 2,69 \\
\pm 2,65 \\
\pm 0,01\end{array}$ \\
\hline $\begin{array}{l}\text { Diâmetro } \\
\text { mínimo } \\
(\text { Dmin }) \\
(\mathrm{cm})\end{array}$ & $\begin{array}{c}1.399-650 \\
649-300 \\
<300\end{array}$ & $\begin{array}{l}D \min _{2}=0,004923+0,817199 \overline{\mathrm{D}}_{2}+0,33822 \mathrm{Hd}_{2}-2,279692 \sqrt{\mathrm{S}^{2} \mathrm{~d}_{2}}-0,75978 \mathrm{ln}_{2}+0,070966 \mathrm{I}_{2} \\
D \min _{2}=3,589635+1,142362 \overline{\mathrm{D}}_{2}-0,290394 \mathrm{Hd}_{2}-1,638772 \sqrt{\mathrm{S}^{2} \mathrm{~d}_{2}}-1,986395 \ln \mathrm{G}_{2}+0,197052 \mathrm{I}_{2} \\
D \min _{2}=1,748782+1,080354 \overline{\mathrm{D}}_{2}-0,134884 \mathrm{Hd}_{2}-1,834316 \sqrt{\mathrm{S}^{2} \mathrm{~d}_{2}}-0,260461 \ln \mathrm{G}_{2}-0,011404 \mathrm{I}_{2} \\
D \min _{2}=0,004923+0,817199 \overline{\mathrm{D}}_{2}+0,33822 \mathrm{Hd}_{2}-2,279692 \sqrt{\mathrm{S}^{2} \mathrm{~d}_{2}}-0,75978 \ln _{2}+0,070966 \mathrm{I}_{2}\end{array}$ & $\begin{array}{l}80,1 \\
79,5 \\
67,7 \\
67,7\end{array}$ & $\begin{array}{l} \pm 1,49 \\
\pm 1,58 \\
\pm 2,09 \\
\pm 2,09\end{array}$ \\
\hline
\end{tabular}

Índice $1 \mathrm{em}$ todos os atributos $=$ presente.

Índice $2 \mathrm{em}$ todos os atributos $=$ futuro ou idade de prognose. 

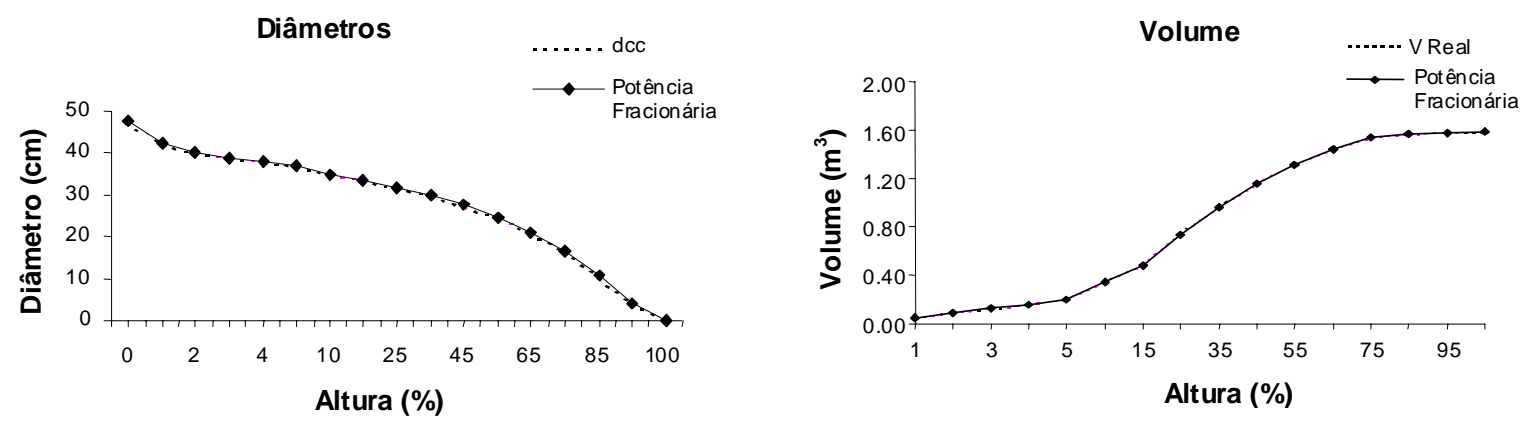

Figura 1 - Perfis referentes a média dos diâmetros das árvores cubadas (a) e ao volume destas (b).

Figure 1 - Cubed trees average diameter (a) and stem volume (b).

Quadro 10 - Parâmetros estimados e medidas de precisão de algumas expressões de desbaste por tipo, número de árvores e classes de número de árvores para Pinus taeda

Table 10 - Estimated parameters and precision measurements by thinning type, number of trees and number classes for Pinus taeda

\begin{tabular}{|c|c|c|c|c|c|c|}
\hline \multirow{2}{*}{$\begin{array}{c}\text { Tipo de } \\
\text { desbaste }^{1 /}\end{array}$} & \multicolumn{2}{|c|}{ Condição } & \multicolumn{2}{|c|}{ Parâmetro } & \multirow[b]{2}{*}{$\mathrm{R}^{2}$} & \multirow[b]{2}{*}{ Syx } \\
\hline & $\begin{array}{l}\text { Número de } \\
\text { árvores }^{2 !}\end{array}$ & Idade $^{3 /}$ & $\beta_{0}$ & $\beta_{1}$ & & \\
\hline 5 & $<1.600$ & $\geq 4 \leq 8$ & $-0,59258496$ & 1,14600925 & 78,59 & 0,02164 \\
\hline 6 & $<1.500$ & $\leq 14$ & $-0,67368634$ & 0,90829984 & 78,28 & 0,020409 \\
\hline 6 & $<1.500$ & $\geq 15 \mathrm{e} \leq 17$ & $-0,62955922$ & 0,92717049 & 70,59 & 0,027873 \\
\hline 6 & $<1.500$ & $\geq 18$ e $\leq 20$ & $-0,18614847$ & 4,0593605 & 99,9 & 0,0001674 \\
\hline 6 & $<1.500$ & $\geq 21$ & $-0,29136948$ & 1,15596397 & 87,10 & 0,008840 \\
\hline 2 & $<600$ & $\leq 22$ & $-0,49935306$ & 1,81510482 & 73,27 & 0,026582 \\
\hline 2 & $<600$ & $\geq 22 \mathrm{e} \leq 23$ & $-0,66438434$ & 2,43794218 & 95,68 & 0,006021 \\
\hline 2 & $<600$ & $\geq 24$ & $-0,57709341$ & 1,96474839 & 77,81 & 0,022513 \\
\hline 3 & $<500$ & $\leq 19$ & $-1,16846881$ & 2,11173603 & 86,62 & 0,013186 \\
\hline 3 & $<500$ & $\geq 20$ e $\leq 22$ & $-0,75221533$ & 1,58485409 & 88,94 & 0,013510 \\
\hline 3 & $<500$ & $\geq 23$ & $-0,73873283$ & 1,85349919 & 75,70 & 0,02613 \\
\hline 3 & $<400$ & --- & $-0,96824032$ & 1,36289769 & 37,18 & 0,065629 \\
\hline 3 & $<400$ & --- & $-0,92415067$ & 0,94448626 & 54,53 & 0,0250061 \\
\hline 3 & $<400$ & --- & $-0,26074486$ & 1,55101998 & 72,82 & 0,0154731 \\
\hline
\end{tabular}

${ }^{1 /}$ Os desbastes dos tipos 5, 6, 2 e 3 correspondem, respectivamente, a: desbaste pré-comercial, $1^{\circ}$ desbaste (sistemático na $6^{\underline{a}}$ linha + seletivo), $2^{\circ}$ desbaste (somente seletivo) e $3^{\circ}$ desbaste (somente seletivo); ${ }^{2 \prime}$ número de árvores para as quais as estimativas são válidas; e ${ }^{3}$ classes de idade para as quais as estimativas são válidas.

Ao considerar o preço da madeira desramada igual ao da não-desramada, a situação que proporcionou a maior rentabilidade nas classes de sítios I e II foi o plantio de 833 árvores/ha (3x4 m). Já na classe de sítio III, a maior rentabilidade foi obtida com o plantio de 1.111 árvores/ha $(3 \times 3 \mathrm{~m})$, o que mostra que em sítios mais produtivos a melhor estratégia tende a ser plantar em espaçamentos mais amplos, permitindo que as árvores maximizem o aproveitamento dos nutrientes, da água e da luz oferecidos pelo local. À medida que a produtividade dos sítios decresce, a tendência é que os plantios sejam feitos em espaçamentos mais reduzidos, para que haja sua melhor ocupação. Embora ocorra aumento dos custos com a redução do espaçamento (mudas, adubos 
etc.), proporcionalmente o volume aumenta em maior intensidade, o que é traduzido no maior valor de VET para estas situações.

Para servir de referência para as empresas que não possuem capital disponível para aquisição de terras ou para aquelas que já possuem terras arrendadas, apresentam-se no Quadro 11 os regimes de manejo mais viáveis economicamente para a situação em que as terras usadas pela empresa são arrendadas.

Para a taxa de desconto de $6 \%$ a.a., a prática da desrama gera lucro mesmo que o preço da madeira desramada seja igual ao da madeira não-desramada. $\mathrm{O}$ regime de manejo mais lucrativo teve um VET de $\mathrm{R} \$ 3.977,12$ quando na simulação considerou-se que o preço da madeira desramada é igual ao da não-desramada. Havendo acréscimo de $40 \%$ no preço da madeira desramada em relação ao da não-desramada, o VET sobe para $\mathrm{R} \$ 6.468,87$, ou seja, o lucro aumenta em $62,6 \%$.
Taxas de desconto altas tendem a inviabilizar economicamente os regimes de manejo, principalmente os das classes de sítios menos produtivos. Por exemplo, na classe de sítio III, a uma taxa de desconto de $8 \%$ a.a., o regime de manejo com um desbaste pré-comercial e um desbaste comercial e com a prática da desrama só será lucrativo se o preço da madeira desramada for $40 \%$ superior ao da não-desramada. Já para a taxa de $10 \%$ é necessário um acréscimo maior que $40 \%$ no preço da madeira desramada para que a atividade seja viável economicamente.

O Quadro 12 mostra os regimes de manejo mais lucrativos para a situação em que as terras usadas pertencem à empresa. Os efeitos da taxa de desconto, do índice de sítio e da variação no preço da madeira são semelhantes aos observados no Quadro 11.

Comparando os Quadros 11 e 12, nota-se que os regimes de manejo implantados em terras próprias são

Quadro 11 - Regimes de manejo economicamente mais rentáveis para o cenário 1, considerando a implantação de Pinus taeda em terras arrendadas

Table 11 - Most profitable management regimes for scenario 1, for Pinus taeda plantation in rented land

\begin{tabular}{|c|c|c|c|c|c|c|c|c|c|c|c|c|c|c|c|}
\hline \multirow{2}{*}{$\begin{array}{l}\text { Preço da } \\
\text { Madeira }\end{array}$} & \multirow{2}{*}{ DI } & \multirow{2}{*}{$S$} & \multirow{2}{*}{$\mathrm{I}_{1}$} & \multirow{2}{*}{ NR } & \multirow{2}{*}{$\mathrm{I}_{2}$} & \multirow{2}{*}{ NR } & \multirow{2}{*}{$\mathrm{CF}$} & \multirow{2}{*}{$\mathrm{VC} 1$} & \multirow{2}{*}{$\mathrm{VC} 2$} & \multirow{2}{*}{ VC3 } & \multirow{2}{*}{ VC4 } & \multirow{2}{*}{ VT } & \multicolumn{3}{|c|}{ VET } \\
\hline & & & & & & & & & & & & & $6 \%$ & $8 \%$ & $10 \%$ \\
\hline \multirow{9}{*}{$\begin{array}{l}\text { Igual ao } \\
\text { da } \\
\text { madeira } \\
\text { não } \\
\text { desramada }\end{array}$} & 833 & 28,5 & 4 & 500 & 12 & 200 & 20 & 40 & 65 & 197 & 547 & 857 & $3.977,12$ & $1.637,36$ & 394,27 \\
\hline & 833 & 28,5 & 4 & 500 & 12 & 200 & 21 & 40 & 65 & 197 & 593 & 901 & $3.956,46$ & $1.569,59$ & 314,35 \\
\hline & 833 & 28,5 & 4 & 500 & 10 & 200 & 20 & 39 & 63 & 172 & 554 & 836 & $3.905,08$ & $1.600,66$ & 378,68 \\
\hline & 833 & 25,5 & 4 & 500 & 8 & 200 & 20 & 33 & 57 & 113 & 446 & 657 & $1.875,60$ & 340,16 & $-452,33$ \\
\hline & 833 & 25,5 & 4 & 550 & 10 & 200 & 21 & 36 & 70 & 158 & 450 & 720 & $1.862,86$ & 299,07 & $-501,56$ \\
\hline & 833 & 25,5 & 4 & 500 & 8 & 200 & 21 & 33 & 57 & 112 & 487 & 696 & $1.860,81$ & 282,79 & $-521,07$ \\
\hline & 1.111 & 22,5 & 4 & 500 & 12 & 200 & 20 & 33 & 59 & 159 & 309 & 575 & 204,87 & $-702,34$ & $-1.148,89$ \\
\hline & 1.111 & 22,5 & 4 & 500 & 12 & 200 & 21 & 33 & 59 & 159 & 338 & 603 & 199,72 & $-739,59$ & $-1.195,30$ \\
\hline & 1.111 & 22,5 & 4 & 500 & 12 & 200 & 22 & 33 & 58 & 158 & 367 & 632 & 169,30 & $-791,56$ & $-1.250,17$ \\
\hline \multirow{9}{*}{$\begin{array}{l}\text { Com } \\
\text { acrésc. } \\
\text { de } 40 \%\end{array}$} & 833 & 28,5 & 4 & 500 & 12 & 200 & 20 & 40 & 65 & 197 & 547 & 857 & $6.468,87$ & $3.189,69$ & $1.423,51$ \\
\hline & 833 & 28,5 & 4 & 600 & 8 & 200 & 20 & 42 & 76 & 125 & 559 & 816 & $6.414,27$ & $3.200,32$ & $1.471,20$ \\
\hline & 833 & 28,5 & 4 & 550 & 8 & 200 & 20 & 40 & 68 & 126 & 569 & 815 & $6.411,58$ & $3.184,73$ & $1.450,35$ \\
\hline & 833 & 25,5 & 4 & 500 & 8 & 200 & 20 & 33 & 57 & 113 & 446 & 657 & $4.290,37$ & $1.859,12$ & 567,22 \\
\hline & 833 & 25,5 & 4 & 550 & 10 & 300 & 20 & 37 & 74 & 172 & 424 & 715 & $4.267,42$ & $1.756,69$ & 431,91 \\
\hline & 833 & 25,5 & 4 & 550 & 10 & 250 & 20 & 37 & 71 & 164 & 418 & 698 & $4.265,62$ & $1.801,64$ & 495,41 \\
\hline & 833 & 22,5 & 4 & 500 & 12 & 300 & 20 & 29 & 63 & 165 & 318 & 590 & $2.360,90$ & 572,42 & $-351,87$ \\
\hline & 833 & 25,5 & 4 & 500 & 12 & 250 & 20 & 28 & 61 & 160 & 312 & 578 & $2.342,48$ & 589,81 & $-319,69$ \\
\hline & 833 & 25,5 & 4 & 500 & 10 & 250 & 20 & 28 & 58 & 136 & 315 & 552 & $2.273,40$ & 560,31 & $-327,98$ \\
\hline
\end{tabular}

DI = densidade inicial de plantio (árvores/ha); S = índice de sítio (m); $\mathrm{I}_{1}=$ idade do desbaste pré-comercial (anos); NR = número de árvores remanescentes; $\mathrm{I}_{2}=$ Idade do $1^{\underline{0}}$ desbaste comercial; $\mathrm{CF}=$ idade do corte final; $\mathrm{VC} 1$ = volume comercial com diâmetro entre 8 e $17,9 \mathrm{~cm} ; \mathrm{VC} 2=$ volume comercial com diâmetro entre 18 e $24,9 \mathrm{~cm}$; VC3 = volume comercial com diâmetro entre 25 e $34,9 \mathrm{~cm}$; VC4 = volume comercial com diâmetro maior ou igual a $35 \mathrm{~cm}$; e VT = volume total. 
bem mais lucrativos que os implantados em terras arrendadas. Por exemplo, para a taxa de desconto de $6 \%$ a.a. e preço da madeira desramada $40 \%$ superior ao da nãodesramada, o VET do melhor regime de manejo é de $\mathrm{R} \$ 6.468,87$ se o plantio for em terras arrendadas e de $\mathrm{R} \$ 8.956,91$ se ele for em terras próprias.

\subsection{Análise Econômica dos Regimes de Manejo Gerados para o Cenário 2}

Ao analisar os 31.104 regimes de manejo gerados para o cenário 2, verificou-se que nas classes de sítios I e II o VET por hectare situou-se nas faixas de $\mathrm{R} \$ 1.500,00$ a $\mathrm{R} \$ 11.510,00$ e de $\mathrm{R} \$ 50,00$ a $\mathrm{R} \$ 8.300,00$, respectivamente. Já na classe de sítio III, em situações em que o preço da madeira desramada é igual ao preço da madeira não-desramada, há regimes de manejo com VET negativo. Contudo, basta elevar o preço da madeira desramada em $40 \%$ para que todos os cenários de regimes de manejo simulados para aquela classe de sítio se tornem lucrativos.

O espaçamento $3 \times 4 \mathrm{~m}$ foi o que proporcionou a maior rentabilidade para as três classes de sítio consideradas. Este fato já era esperado para as classes de sítio I e II, uma vez que quando são realizados desbastes em sítios mais produtivos as árvores aceleram o ritmo de crescimento em diâmetro. Contudo, nas classes de sítio III, contrariamente ao esperado, o espaçamento que mais se destacou também foi o $3 \times 4 \mathrm{~m}$. Este fato provavelmente ocorreu em face de os custos de realizar um desbaste pré-comercial e dois desbastes comerciais em um número maior de indivíduos terem sido proporcionalmente superiores ao volume gerado para fins mais nobres de uso da madeira.

Quadro 12 - Regimes de manejo economicamente mais rentáveis para o cenário 1, considerando a implantação de Pinus taeda em terras da própria empresa

Table 12 - Most profitable management regimes for scenario 1, for Pinus taeda plantation in land owned by the company

\begin{tabular}{|c|c|c|c|c|c|c|c|c|c|c|c|c|c|c|c|}
\hline \multirow{2}{*}{$\begin{array}{l}\text { Preço da } \\
\text { Madeira }\end{array}$} & \multirow{2}{*}{ I } & & \multirow{2}{*}{$\mathrm{I}_{1}$} & \multirow{2}{*}{$\mathrm{R}$} & \multirow{2}{*}{$\mathrm{I}_{2}$} & \multirow{2}{*}{ IR } & \multirow{2}{*}{$F$} & \multirow{2}{*}{ VC1 } & \multirow{2}{*}{$\mathrm{VC} 2$} & \multirow{2}{*}{ VC3 } & \multirow{2}{*}{ VC4 } & \multirow{2}{*}{ VT } & \multicolumn{3}{|c|}{ VET } \\
\hline & & & & & & & & & & & & & $6 \%$ & $8 \%$ & $10 \%$ \\
\hline \multirow{9}{*}{$\begin{array}{l}\text { Igual ao } \\
\text { da } \\
\text { madeira } \\
\text { não } \\
\text { desramada }\end{array}$} & 833 & 3,5 & 4 & 500 & 12 & 200 & 20 & 40 & 65 & 197 & 547 & 857 & $5.842,21$ & $.237,28$ & 289,10 \\
\hline & 833 & 28,5 & 4 & 500 & 12 & 200 & 21 & 40 & 5 & 197 & 593 & 901 & $5.810,93$ & $.145,13$ & 181,70 \\
\hline & 833 & 28,5 & 4 & 500 & 10 & 200 & 20 & 39 & 63 & 172 & 554 & 836 & $5.778,61$ & $2.214,27$ & 289,90 \\
\hline & 833 & 25,5 & 4 & 550 & 10 & 200 & 21 & 36 & 70 & 158 & 450 & 720 & $3.339,34$ & 757,65 & $-601,520$ \\
\hline & 833 & 25,5 & 4 & 600 & 10 & 200 & 21 & 38 & 80 & 158 & 437 & 721 & $3.321,67$ & 769,59 & $-581,42$ \\
\hline & 833 & 25,5 & 4 & 550 & 10 & 200 & 20 & 36 & 71 & 158 & 410 & 683 & $3.320,28$ & 812,15 & $-527,00$ \\
\hline & 1.111 & 22,5 & 4 & 500 & 12 & 200 & 20 & 33 & 59 & 159 & 309 & 575 & $1.372,32$ & $-313,76$ & $-1.194,10$ \\
\hline & 1.111 & 22,5 & 4 & 500 & 12 & 200 & 21 & 33 & 59 & 159 & 338 & 603 & $1.361,40$ & $-365,74$ & $-1.257,36$ \\
\hline & 1.111 & 22,5 & 4 & 500 & 12 & 200 & 22 & 33 & 58 & 158 & 367 & 632 & $1.316,62$ & $-437,38$ & $-1.331,90$ \\
\hline \multirow{9}{*}{$\begin{array}{c}\text { Com } \\
\text { acrésc. } \\
\text { de } 40 \%\end{array}$} & 833 & 28,5 & 4 & 500 & 12 & 200 & 20 & 40 & 65 & 197 & 547 & 857 & $8.956,91$ & $4.177,69$ & $1.575,65$ \\
\hline & 833 & 28,5 & 4 & 600 & 8 & 200 & 20 & 42 & 76 & 125 & 559 & 816 & $8.914,23$ & $4.218,29$ & $1.662,70$ \\
\hline & 1.111 & 28,5 & 4 & 550 & 8 & 200 & 20 & 50 & 71 & 132 & 565 & 827 & $8.911,53$ & $4.192,26$ & $1.629,18$ \\
\hline & 833 & 25,5 & 4 & 500 & 8 & 200 & 20 & 33 & 57 & 113 & 446 & 657 & $6.336,60$ & $2.695,47$ & 729,90 \\
\hline & 833 & 25,5 & 4 & 550 & 10 & 250 & 20 & 37 & 71 & 164 & 418 & 698 & $6.296,06$ & $2.610,66$ & 625,95 \\
\hline & 833 & 25,5 & 4 & 550 & 10 & 200 & 20 & 36 & 71 & 158 & 410 & 683 & $6.286,31$ & $2.666,21$ & 707,84 \\
\hline & 833 & 22,5 & 4 & 500 & 12 & 300 & 20 & 29 & 63 & 165 & 318 & 590 & $3.993,12$ & $1.223,65$ & $-242,16$ \\
\hline & 833 & 22,5 & 4 & 500 & 12 & 250 & 20 & 28 & 61 & 160 & 312 & 578 & $3.989,01$ & $1.260,48$ & $-206,09$ \\
\hline & 1.111 & 22,5 & 4 & 500 & 12 & 200 & 20 & 33 & 59 & 159 & 309 & 575 & $3.903,99$ & $1.251,15$ & $-165,29$ \\
\hline
\end{tabular}

$\mathrm{DI}=$ densidade inicial de plantio (árvores/ha); $\mathrm{S}=$ índice de sítio $(\mathrm{m}) ; \mathrm{I}_{1}=$ idade do desbaste pré-comercial (anos); NR = número de árvores remanescentes; $\mathrm{I}_{2}=$ idade do $1^{\circ}$ desbaste comercial; $\mathrm{CF}=$ idade do corte final; $\mathrm{VC} 1=$ volume comercial com diâmetro entre $8 \mathrm{e}$ $17,9 \mathrm{~cm} ; \mathrm{VC} 2$ = volume comercial com diâmetro entre 18 e $24,9 \mathrm{~cm} ; \mathrm{VC} 3$ = volume comercial com diâmetro entre 25 e $34,9 \mathrm{~cm} ; \mathrm{VC} 4=$ volume comercial com diâmetro maior ou igual a $35 \mathrm{~cm}$; VT = volume total. 
Quadro 13 - Regimes de manejo economicamente mais rentáveis para o cenário 2, considerando a implantação de Pinus taeda em terras arrendadas

Table 13 - Most profitable management regimes for scenario 2, for Pinus taeda plantation in rented land

\begin{tabular}{|c|c|c|c|c|c|c|c|c|c|c|c|c|c|c|c|c|c|}
\hline \multirow{2}{*}{$\begin{array}{l}\text { Preço da } \\
\text { Madeira }\end{array}$} & \multirow{2}{*}{ DI } & \multirow{2}{*}{ S } & \multirow{2}{*}{$\mathrm{I}_{1}$} & \multirow{2}{*}{ NR } & \multirow{2}{*}{$\mathrm{I}_{2}$} & \multirow{2}{*}{ NR } & \multirow{2}{*}{$\mathrm{I}_{3}$} & \multirow{2}{*}{ NR } & \multirow{2}{*}{$\mathrm{CF}$} & \multirow{2}{*}{$\mathrm{VC} 1$} & \multirow{2}{*}{$\mathrm{VC} 2$} & \multirow{2}{*}{ VC3 } & \multirow{2}{*}{ VC4 } & \multirow{2}{*}{ VT } & \multicolumn{3}{|c|}{ VET } \\
\hline & & & & & & & & & & & & & & & $6 \%$ & $8 \%$ & $10 \%$ \\
\hline \multirow{9}{*}{$\begin{array}{c}\text { Igual ao } \\
\text { da } \\
\text { madeira } \\
\text { não } \\
\text { desramada }\end{array}$} & 833 & 28,5 & 4 & 50 & 12 & 400 & 14 & 100 & 21 & 40 & 64 & 189 & 578 & 892 & $4.602,18$ & $.048,44$ & 81,66 \\
\hline & 833 & 8,5 & 4 & 00 & 12 & 3( & 4 & 100 & 21 & 38 & 63 & 193 & 570 & 881 & $4.541,10$ & $.016,19$ & 65,23 \\
\hline & 1.111 & 28,5 & 4 & 500 & 12 & 400 & 14 & 100 & 21 & 49 & 65 & 196 & 579 & 904 & $4.521,49$ & $1.982,25$ & 24,83 \\
\hline & 833 & 25,5 & 4 & 500 & 12 & 400 & 14 & 100 & 21 & 34 & 62 & 181 & 428 & 716 & $2.476,58$ & 744,88 & $-167,27$ \\
\hline & 833 & 25,5 & 4 & 500 & 12 & 300 & 14 & 100 & 21 & 34 & 61 & 175 & 425 & 706 & $2.417,70$ & 711,75 & $-185,97$ \\
\hline & 833 & 25,5 & 4 & 500 & 12 & 400 & 14 & 100 & 20 & 34 & 62 & 182 & 394 & 684 & $2.387,97$ & 722,27 & $-161,33$ \\
\hline & 833 & 22,5 & 4 & 500 & 12 & 300 & 15 & 100 & 21 & 28 & 60 & 153 & 289 & 547 & 169,56 & $-694,31$ & $1.122,09$ \\
\hline & 833 & 22,5 & 4 & 500 & 12 & 400 & 15 & 100 & 21 & 28 & 61 & 166 & 288 & 560 & 143,35 & $-719,79$ & $-1.146,22$ \\
\hline & 833 & 22,5 & 4 & 500 & 12 & 300 & 15 & 100 & 20 & 28 & 60 & 154 & 267 & 526 & 127,91 & $-698,22$ & $1.110,56$ \\
\hline \multirow{9}{*}{$\begin{array}{c}\text { Com } \\
\text { acrésc. } \\
\text { de } 40 \%\end{array}$} & 833 & 28,5 & 4 & 500 & 12 & 400 & 14 & 100 & 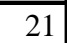 & 40 & 64 & 189 & \begin{tabular}{|l|}
578 \\
\end{tabular} & 892 & $3.081,85$ & $4.259,48$ & 177,35 \\
\hline & 833 & 28,5 & 4 & 500 & 12 & 400 & 14 & 100 & 20 & 40 & 65 & 190 & 533 & 849 & $8.013,92$ & $4.267,90$ & $2.215,40$ \\
\hline & 1.111 & 28,5 & 4 & 500 & 12 & 400 & 14 & 100 & 21 & 49 & 65 & 196 & 579 & 904 & $7.889,94$ & $4.120,54$ & $2.069,91$ \\
\hline & 833 & 25,5 & 4 & 500 & 12 & 400 & 14 & 100 & 21 & 34 & 62 & 181 & 428 & 716 & $5.400,24$ & $2.599,98$ & $1.085,84$ \\
\hline & 833 & 25,5 & 4 & 500 & 12 & 400 & 14 & 100 & 20 & 34 & 62 & 182 & 394 & 684 & $5.381,45$ & $2.626,57$ & $1.127,94$ \\
\hline & 1.111 & 25,5 & 4 & 500 & 12 & 400 & 14 & 100 & 21 & 40 & 63 & 176 & 429 & 726 & $5.285,75$ & $2.514,68$ & $1.018,42$ \\
\hline & 833 & 22,5 & 4 & 500 & 12 & 400 & 15 & 100 & 20 & 28 & 61 & 167 & 265 & 539 & $2.557,64$ & 823,65 & $-97,87$ \\
\hline & 833 & 22,5 & 4 & 500 & 12 & 400 & 15 & 100 & 21 & 28 & 61 & 166 & 288 & 560 & $2.555,45$ & 795,77 & $-133,27$ \\
\hline & 833 & 22,5 & 4 & 500 & 12 & 300 & 15 & 100 & 20 & 28 & 60 & 154 & 267 & 526 & $2.452,06$ & 770,35 & $-123,27$ \\
\hline
\end{tabular}

$\mathrm{DI}=$ densidade inicial de plantio (árvores/ha); $\mathrm{S}=$ índice de sítio $(\mathrm{m}) ; \mathrm{I}_{1}=$ idade do desbaste pré-comercial $($ anos); NR = número de árvores remanescentes; $\mathrm{I}_{2}=$ idade do $1^{\mathrm{o}}$ desbaste comercial; $\mathrm{I}_{3}=$ idade do $2^{\mathrm{o}}$ desbaste comercial; $\mathrm{CF}=$ idade do corte final; VC1 = volume comercial com diâmetro entre 8 e $17,9 \mathrm{~cm}$; VC2 = volume comercial com diâmetro entre 18 e $24,9 \mathrm{~cm}$; VC $3=$ volume comercial com diâmetro entre 25 e $34,9 \mathrm{~cm}$; VC4 = volume comercial com diâmetro maior ou igual a $35 \mathrm{~cm}$; e VT = volume total.

O Quadro 13 mostra os regimes de manejo economicamente mais rentáveis para o cenário 2, quando o plantio é feito em terras arrendadas. Para o regime mais lucrativo e usando a taxa de desconto de $6 \%$ a.a. como referência, o VET aumenta em $\mathrm{R} \$ 3.479,67$ (passa de $\mathrm{R} \$ 4.602,18$ para $\mathrm{R} \$ 8.081,85$ ) quando o preço da madeira desramada é $40 \%$ superior ao preço da madeira não-desramada. Tal fato evidencia que investir em técnicas que melhorem a qualidade da madeira é uma opção interessante do ponto de vista econômico.

Os regimes de manejo para as florestas implantadas em terras próprias (Quadro 14) são nitidamente superiores aos das florestas implantadas em terras arrendadas (Quadro 13), em todas as condições de sítio, preço da madeira e taxa de desconto simuladas. Por exemplo, a uma taxa de $6 \%$ a.a., na situação em que o preço da madeira desramada é $40 \%$ superior ao preço da madeira não-desramada, o VET é de R $\$ 8.081,85$ quando as terras são arrendadas e de $\mathrm{R} \$ 11.008,65$ quando elas pertencem à empresa.

É nítida a supremacia dos regimes de manejo implantados nas classes de sítios I e II em relação aos da classe de sítio III, evidenciando que preferencialmente o manejo conjugado desbaste/ desrama deve ser implementado em sítios de alta produtividade, uma vez que nesses sítios ocorre maior resposta em termos de crescimento em área basal das árvores remanescentes após a realização de cada desbaste.

Comparando os resultados dos dois cenários, constata-se que o cenário 2 é mais viável economicamente que o cenário 1 , em todas as situações analisadas. Por exemplo, nas simulações em que a terra pertence à própria empresa, considerando-se a taxa de desconto de $8 \%$ a.a. e o preço da madeira desramada $40 \%$ superior ao da madeira não-desramada, o VET do melhor regime de manejo simulado é de $\mathrm{R} \$ 4.177,69$ para o cenário 1 (Quadro 12) e de $\mathrm{R} \$ 5.537,32$ para o cenário 2 (Quadro 14).

R. Árvore, Viçosa-M G, v.26, n.6, p.699-713, 2002 
Quadro 13 - Regimes de manejo economicamente mais rentáveis para o cenário 2, considerando a implantação de Pinus taeda em terras arrendadas

Table 13 - Most profitable management regimes for scenario 2, for Pinus taeda plantation in rented land

\begin{tabular}{|c|c|c|c|c|c|c|c|c|c|c|c|c|c|c|c|c|c|}
\hline \multirow{2}{*}{\begin{tabular}{|l} 
Preço da \\
Madeira \\
\end{tabular}} & \multirow{2}{*}{ DI } & \multirow{2}{*}{$\mathrm{S}$} & \multirow{2}{*}{$\mathrm{I}_{1}$} & \multirow{2}{*}{ NR } & \multirow[b]{2}{*}{12} & \multirow{2}{*}{$\mathrm{IR}$} & \multirow[t]{2}{*}{$I_{-}$} & \multirow{2}{*}{ NR } & \multirow{2}{*}{$\mathrm{CF}$} & \multirow{2}{*}{ VC1 } & \multirow{2}{*}{ VC2 } & \multirow{2}{*}{ VC3 } & \multirow{2}{*}{ VC4 } & \multirow{2}{*}{ VT } & \multicolumn{3}{|c|}{ VET } \\
\hline & & & & & & & & & & & & & & & $6 \%$ & $8 \%$ & $0 \%$ \\
\hline \multirow{9}{*}{$\begin{array}{c}\text { Igual ao } \\
\text { da } \\
\text { madeira } \\
\text { não } \\
\text { desramada }\end{array}$} & 833 & 28,5 & 4 & 0 & 12 & 00 & 14 & 100 & 21 & 40 & 64 & 189 & 578 & 892 & $4.602,18$ & $.048,44$ & 81,66 \\
\hline & 833 & 28,5 & 4 & & 12 & 0 & 14 & 100 & 21 & 38 & 63 & 93 & 570 & 881 & $4.541,10$ & $.016,19$ & 5,23 \\
\hline & 1.111 & 28,5 & 4 & 500 & 12 & 400 & 14 & 100 & 21 & 49 & 65 & 196 & 579 & 904 & $4.521,49$ & $1.982,25$ & 524,83 \\
\hline & 833 & 25,5 & 4 & 5 & 12 & 400 & 14 & 100 & 21 & 34 & 62 & 181 & 428 & 716 & $2.476,58$ & 38 & $-167,27$ \\
\hline & 833 & 25,5 & 4 & 5 & 12 & 30 & 14 & 100 & 21 & 34 & 61 & 175 & 425 & 706 & $2.417,70$ & 711,75 & $-185,97$ \\
\hline & 833 & 25,5 & 4 & 500 & 12 & 400 & 14 & 100 & 20 & 34 & 62 & 182 & 394 & 684 & $.381,91$ & 27 & $-161,33$ \\
\hline & 833 & 22,5 & 4 & 500 & 12 & 300 & 15 & 100 & 21 & 28 & 60 & 153 & 289 & 547 & 169,56 & $-694,31$ & $-1.122,09$ \\
\hline & 833 & 22,5 & 4 & 500 & 12 & 400 & 15 & 100 & 21 & 28 & 61 & 166 & 288 & 560 & 35 & -71 & $-1.146,22$ \\
\hline & 833 & 22,5 & 4 & 50 & 12 & 30 & 15 & 100 & 20 & 28 & 60 & 154 & 267 & 52 & 91 & 22 & $.110,56$ \\
\hline \multirow{9}{*}{$\begin{array}{c}\text { Com } \\
\text { acrésc. } \\
\text { de } 40 \%\end{array}$} & 83. & 28,5 & 4 & 5 & 12 & 40 & 14 & 10 & 21 & 40 & 64 & 189 & 578 & 892 & $3.081,85$ & 4.25 & $2.177,35$ \\
\hline & 833 & 28,5 & 4 & 500 & 12 & 400 & 14 & 100 & 20 & 40 & 65 & 190 & 533 & 849 & $8.013,92$ & $4.267,90$ & $2.215,40$ \\
\hline & 1.111 & 28,5 & 4 & 500 & 12 & 400 & 14 & 100 & 21 & 49 & 65 & 196 & 579 & 904 & $7.889,94$ & $4.120,54$ & $2.069,91$ \\
\hline & 833 & 25,5 & 4 & 500 & 12 & 400 & 14 & 100 & 21 & 34 & 62 & 181 & 428 & 716 & $5.400,24$ & $2.599,98$ & $1.085,84$ \\
\hline & 833 & 25,5 & 4 & 500 & 12 & 400 & 14 & 100 & 20 & 34 & 62 & 182 & 394 & 684 & $5.381,45$ & $2.626,57$ & $1.127,94$ \\
\hline & 1.111 & 25,5 & 4 & 500 & 12 & 400 & 14 & 100 & 21 & 40 & 63 & 176 & 429 & 726 & $5.285,75$ & $2.514,68$ & $1.018,42$ \\
\hline & 833 & 22,5 & 4 & 500 & 12 & 400 & 15 & 100 & 20 & 28 & 61 & 167 & 265 & 539 & $2.557,64$ & 823,65 & $-97,87$ \\
\hline & 833 & 22,5 & 4 & 500 & 12 & 400 & 15 & 100 & 21 & 28 & 61 & 166 & 288 & 560 & $2.555,45$ & 795,77 & $-133,27$ \\
\hline & 833 & 22,5 & 4 & 500 & 12 & 300 & 15 & 100 & 20 & 28 & 60 & 154 & 267 & 526 & $2.452,06$ & 770,35 & $-123,27$ \\
\hline
\end{tabular}

$\mathrm{DI}=$ densidade inicial de plantio (árvores/ha); $\mathrm{S}=$ índice de sítio $(\mathrm{m}) ; \mathrm{I}_{1}=$ idade do desbaste pré-comercial (anos); NR = número de árvores remanescentes; $\mathrm{I}_{2}=$ idade do $1^{\circ}$ desbaste comercial; $\mathrm{I}_{3}=$ idade do $2^{\underline{o}}$ desbaste comercial; $\mathrm{CF}=$ idade do corte final; VC1 = volume comercial com diâmetro entre 8 e $17,9 \mathrm{~cm}$; VC2 = volume comercial com diâmetro entre 18 e $24,9 \mathrm{~cm}$; VC3 = volume comercial com diâmetro entre 25 e $34,9 \mathrm{~cm}$; VC4 = volume comercial com diâmetro maior ou igual a $35 \mathrm{~cm}$; e VT = volume total.

\section{CONCLUSÕES}

As principais conclusões deste estudo foram:

- O modelo de crescimento e produção desenvolvido apresenta uma equação de índice de sítio estável, tem equações que expressam os atributos do povoamento precisas e sem tendenciosidade, apresenta eficácia na compatibilidade entre a área basal do povoamento e a obtida da distribuição Weibull e apresentou descritor de desbaste preciso em $98 \%$ das situações testadas, sendo, portanto, um sistema preciso.

- Os regimes de manejo com a realização de um desbaste pré-comercial seguido de dois desbastes comerciais e desrama devem ser adotados, uma vez que eles são mais lucrativos que os regimes com um desbaste précomercial seguido de um desbaste comercial e desrama;
- Preferencialmente, a empresa deve investir em sítios de alta produtividade; no presente caso o estudo indicou os índices de sítio 28,5 e 25,5 na idade de referência de 20 anos, para Pinus taeda;

- Arrendar terras para plantar Pinus taeda é uma opção lucrativa, principalmente se o sítio for de alta produtividade. Contudo, para o estudo em questão o plantio de Pinus taeda em terras próprias é mais lucrativo que o plantio em terras arrendadas.

- A densidade inicial de plantio ideal para os índices de sítio $28,5,25,5$ e 23,5 é de 833 árvores/ha, podendose também adotar 1.111 árvores/ha como alternativa

- As taxas de desconto maiores que $10 \%$ a.a. inviabilizam economicamente a maioria dos regimes de manejo, principalmente os das classes de sítio pouco produtivas. 
- A lucratividade dos regimes de manejo aumenta bastante quando se considera a elevação do preço da madeira, devido à melhoria de sua qualidade promovida pela desrama das árvores.

\section{AGRADECIMENTO}

À Piza Florestal S.A., pelo apoio e fornecimento de dados necessários para a realização deste estudo.

\section{REFERÊNCIAS BIBLIOGRÁFICAS}

BENTLEY, W.; TEENGUARDEN, D. Financial maturity: a theory review. Forest Science, v. 11, n. 3, p. 76-87, 1965.

BURKHART,H. E. Development of empirical growth and yield models. In: EMPIRICAL AND PROCESS-BASED MODELS FOR FOREST TREE AND STAND GROWTH SIMULATION, 1., 1997, Oeiras. Anais... Lisboa: Salamandra, 1999. p. 53-60.

FISCHER, F. Eficiência dos modelos polinomiais e das razões de volume na estimativa volumétrica dos sortimentos e do perfil do fuste de Pinus taeda $\mathrm{L}$. Lavras: UFLA, 1997, 167 p. Dissertação (Mestrado em Engenharia Florestal) - Universidade Federal de Lavras, 1997.

FISCHER, F. et al. Exatidão dos modelos polinomiais nãosegmentados e das razões entre volumes para representar o perfil do tronco de Pinus taeda. Ciência Florestal, v. 11, n. 1, p. 167-188, 2001.

GOMES, F. S. A seleção de regimes de manejo mais rentáveis em Pinus taeda $\mathrm{L}$. na produção de madeira para papel e celulose. Curitiba: UFPR, 1999. 137p.

Dissertação (Mestrado em Ciências Florestais) Universidade Federal do Paraná, 1999.
HRADETZKY, J. Analyse und interpretation statistisher abränger keiten. (Biometrische Beiträge zu aktuellen forschungs projekten). Baden - Württemberg Mitteilungen der FVA, Helf 76, 1976. 146 p. (Abt. Biometric und Informatik, 21).

KIMMINS, J. P. et al. Foreseeing and forecasting the horizon: hybrid simulation modeling of forest ecosystem sustainability. In: EMPIRICAL AND PROCESS-BASED MODELS FOR FOREST TREE AND STAND GROWTH SIMULATION, 1., 1997, Oeiras. Anais... Lisboa: Salamandra,1999. p. 431-442.

MACEDO, A. R. P.; MATTOS, R. L. G. Produtos florestais madeira em tora, madeira serrada e painéis. Panorama 1980/1992. S.1.: BNDES/FINAME/BNDESPAR, 1995. $41 \mathrm{p}$.

ROSOT, M. A. D. Estudo comparativo de métodos para avaliação volumétrica por unidade de área em povoamento de Pinus taeda L. Curitiba: UFPR, 1989. 163 p. Dissertação (Mestrado em Engenharia Florestal) Universidade Federal do Paraná, 1989.

SCOLFORO, J. R. S. O sistema Pinusprog. Lavras: UFLA/FAEPE, 1999a. 69 p.

SCOLFORO, J. R. S. O sistema Invest. Lavras: UFLA/ FAEPE. 1999b. 42 p.

SCOLFORO, J. R. S.; ACERBI JUNIOR, F. W.; ASSIS, A. L. A model for evaluation of forest growth and yield of Pinus taeda with thinning descriptor, in Brazil. Forest Ecology and Management. 2001, 20 p. (submetido)

SOKAL, R. N.; ROHLF, F. J. Biometry. Freeman, San Francisco: 1981. 859 p. 